\title{
Dynamics of Lithium Dendrite Growth and Inhibition - Pulse Charging Experiments and Monte Carlo Calculations
}

Asghar Aryanfar ${ }^{1}$, Daniel Brooks ${ }^{2}$, Agustín J. Colussi ${ }^{1 *}$, Boris V. Merinov², William A. Goddard III', and Michael R. Hoffmann ${ }^{1^{*}}$

'Linde Center for Global Environmental Science, ${ }^{2}$ Materials and Process Simulation Center, California Institute of Technology, California 91125, U.S.A.

\footnotetext{
*Corresponding Authors: AJC: ajcoluss@caltech.edu; MRH: $\underline{\text { mrh@caltech.edu }}$
} 
Abstract: Short-circuiting via dendrites compromises the reliability of Li-metal batteries. Since dendrites ensue from instabilities inherent to electrodeposition they should be amenable to dynamic control. Here we report experiments in a scaled coin cell prototype where the average lengths of dendrite populations were shortened $\sim 2.5$ times upon charging with $1 \mathrm{~ms}$ rectangular pulses followed by $3 \mathrm{~ms}$ rest periods. Monte Carlo simulations dealing with $\mathrm{Li}^{+}$ diffusion and electromigration show that experiments involving 20 ms pulses, in contrast, were ineffective because prolonged migration in the strong electric fields converging to highcurvature dendrite tips generates depleted layers that cannot be replenished by diffusion after $60 \mathrm{~ms}$ rest periods. Since the application of pulses shorter than the characteristic time for capacitively polarizing electric double layers in our system ( $\tau_{\mathrm{C}} \sim 3 \mathrm{~ms}$ ) would approach DC charging, we infer that dendrite propagation may be inhibited (albeit not suppressed) by pulsing at appropriate frequency ranges.

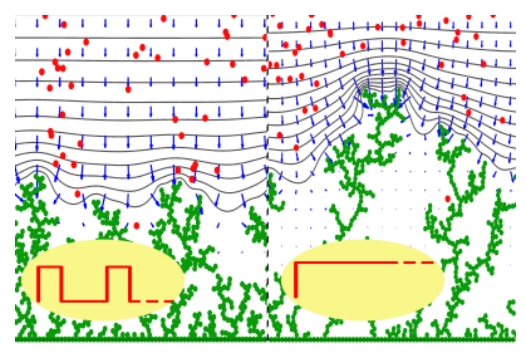

Table of contents graphics

Keywords: Lithium batteries - Short-circuiting - Electromigration - Diffuse charge dynamics - Pulse electrolysis - Depletion double layers 
The specific high energy and power capacities of lithium-metal $\left(\mathrm{Li}^{\circ}\right)$ batteries are ideally suited to power portable devices, and as storage units for intermittent renewable energy sources. ${ }^{1-7}$ $\mathrm{Li}^{0}$, the lightest and most electropositive metal, should be the optimal anode material for rechargeable batteries if it were not for the fact that such devices fail unexpectedly by shortcircuiting via the dendrites that grow across electrodes upon recharging. ${ }^{8-9}$ This phenomenon triggers a series of adverse events that start with overheating, followed by the thermal decomposition and ultimately the ignition of the organic solvents used in such devices. This flaw represents a major safety issue..$^{10-12}$

$\mathrm{Li}^{0}$ dendrites have been imaged, probed and monitored with a wide array of techniques. ${ }^{4-5}$, ${ }^{13}$ Their formation was analyzed ${ }^{14-15}$ and simulated at various levels of realism. ${ }^{9,16-17}$ Numerous empirical and semi-empirical strategies were employed for mitigating the formation of $\mathrm{Li}^{\mathrm{O}}$ dendrites, mostly based on variations of electrode materials and morphologies, and operational conditions. $^{2}$ Thus, there are reports on the effect of current density, ${ }^{18-20}$ electrode surface morphology, ${ }^{10}$ solvent and electrolyte composition, ${ }^{21-24}$ electrolyte concentration, ${ }^{18}$ evolution time, ${ }^{25}$ the use of powder electrodes, ${ }^{26}$ and adhesive lamellar block copolymer barriers ${ }^{27}$ on dendrite growth. Conceivably, further progress in this field could accrue from the deeper insights on the mechanism of dendrite propagation gained by increasingly realistic, and properly designed experiments and modeling calculations. ${ }^{23,}{ }^{28}$ We considered that $\mathrm{Li}^{0}$ dendrite nucleation and propagation are intrinsic to electrodeposition as a dynamic process under nonequilibrium conditions. ${ }^{5,14}$ Also, that in contrast with purely diffusive crystal growth, Li-ion ( $\mathrm{Li}^{+}$) electromigration is an essential feature of electrolytic dendrite growth. ${ }^{29}$ More specifically, we envisioned that runaway dendrite propagation could be slowed by the relaxation of the steep $\mathrm{Li}^{+}$concentration gradients and strong electric field that develop around dendrite tips during charging. This is not a new strategy, ${ }^{30}$ but to our knowledge the quantitative statistical impact of pulses of variable duration on dendrite length has not been reported before. Herein we report experiments on dendrite growth in a scaled coin cell prototype of novel design fitted with $\mathrm{Li}^{0}$ electrodes under rectangular cathodic pulses of variable rest periods and frequencies in the $\mathrm{kHz}$ range. Our cell mimics the geometry and aspect ratio of commercial coin cells but it is 
scaled-up to allow for the visual observation of dendrites. The effects of pulsing on stochastic events such as dendrite nucleation and growth are quantified for the first time on the basis of statistical averages over experimental dendrite length distributions. We also present novel coarse-grained Monte Carlo model calculations which, by dealing explicitly with $\mathrm{Li}^{+}$migration in time-dependent non-uniform electric fields, provide valuable insights into the underlying phenomena. Our findings are expected to guide the implementation of safer charging protocols for commercial batteries.

Our experiments were performed in a manually-fabricated electrolytic cell that provides for in situ observation of the dendrites grown on the perimeter of the electrodes at any stage (Fig. 1). The cell consists of two $\mathrm{Li}^{0}$ foil disc electrodes $(1.59 \mathrm{~cm}$ diameter) separated $0.32 \mathrm{~cm}$ by a transparent acrylic ring. The cell was filled with $0.4 \mathrm{~cm}^{3}$ of $1 \mathrm{M} \mathrm{LiClO}_{4}$ in propylene carbonate (PC) as electrolyte. All operations were conducted in an argon-filled $\left(\mathrm{H}_{2} \mathrm{O}, \mathrm{O}_{2}<0.5 \mathrm{ppm}\right)$ glovebox. Arrays of multiple such cells were simultaneously electrolyzed with trains of $2 \mathrm{~mA} \mathrm{~cm}$ pulses of variable duration: $t_{O N}$, and idle ratios: $\gamma=t_{O F F} / t_{O N}$, generated by a programmable multichannel charger. After the passage of 48 mAh (173 Coulombs) through the cells, the lengths of 45 equidistant dendrites around the cell perimeter were measured through the acrylic separator using a Leica M205FA optical microscope. Since in our cell dendrites propagate unimpeded, i.e. in the absence of a porous separator, our experiments may provide more adverse conditions for controlling dendrite propagation than in actual commercial cells. Further details can be found in Experimental Details, Supporting Information, SI.

The lengths and multiplicities $\left[\lambda_{\mathrm{i}}, p_{\mathrm{i}}\right]$ of the 45 dendrites measured in series of experiments performed at $t_{O N}=1$ and $20 \mathrm{~ms}, \gamma=0$ (DC), 1, 2 and 3, have been divided in 8 bins and are shown as histograms in Fig. S1 (SI). Dendrite lengths typically spanned the $200 \mu \mathrm{m}-3000 \mu \mathrm{m}$ range. Their average length $\alpha$, defined by equation (E 1):

$$
\alpha=\frac{\int p_{i} \lambda_{i}}{\int p_{i}}
$$

represents a figure of merit appropriate (vs. the length of a single dendrite chosen arbitrarily) for appraising the effect of pulsing on the outcome of a stochastic processes such as the inception and propagation of dendrites. The resulting $\alpha$ values, normalized to the largest $\alpha$ in 
each set of experiments, are shown as blue bars as functions of $\gamma$ for $t_{O N}=1$ and 20 ms pulses in Fig. 2. It is immediately apparent that the application of [ $t_{O N}=1 \mathrm{~ms} ; t_{O F F}=3 \mathrm{~ms}$ ] pulse trains reduces average dendrite lengths by $\sim 2.4$ times, whereas $t_{O N}=20 \mathrm{~ms}$ pulses are rather ineffective at any $\gamma$.

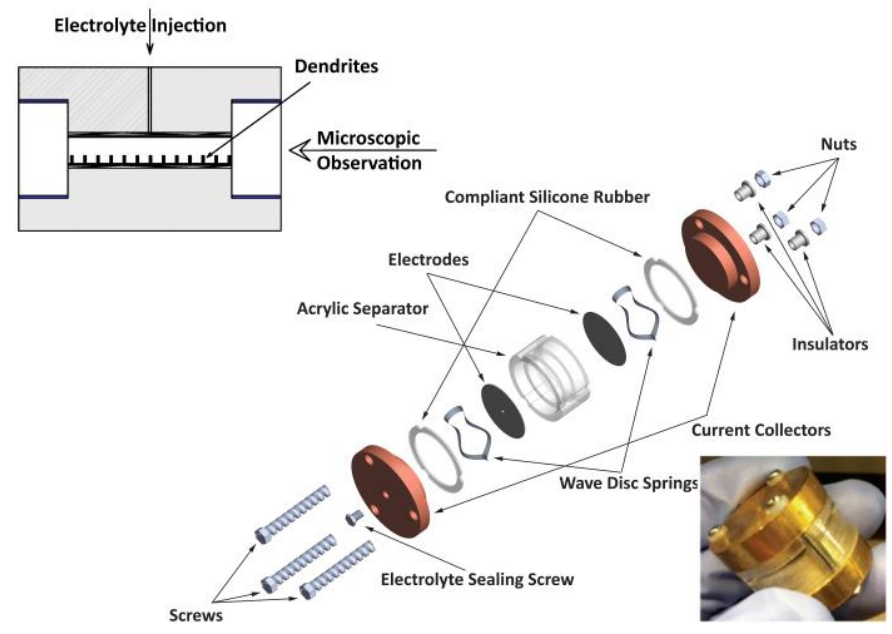

Fig. 1 - Top down: cross section view, exploded view and physical appearance of the cell.

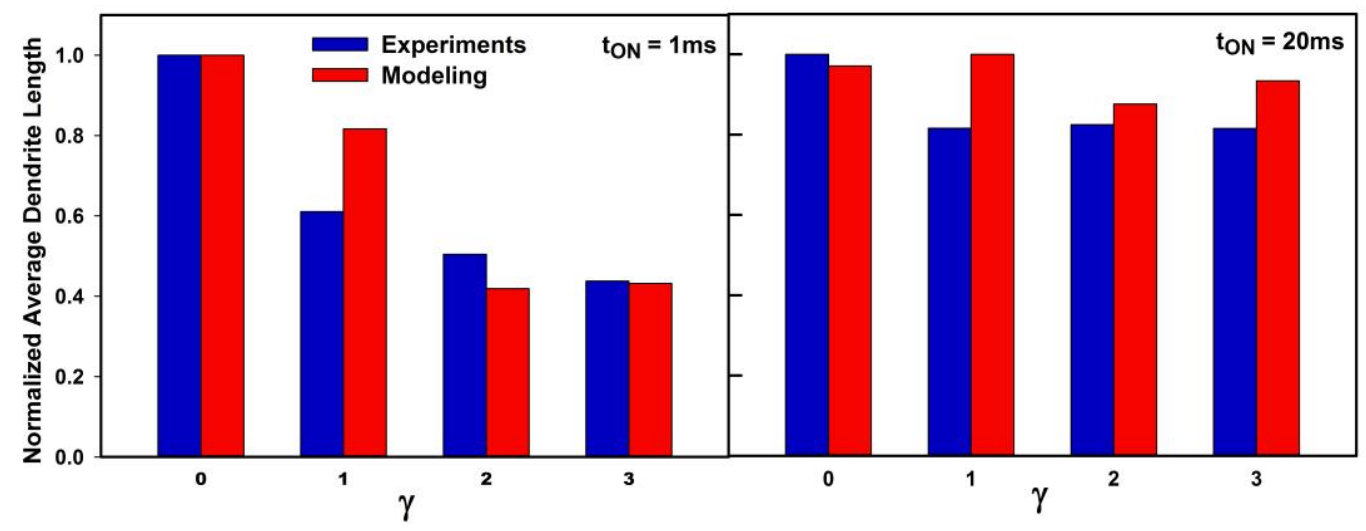

Fig. 2 - Pulse charging effects on the average dendrite length $\alpha$ over a population of 45 dendrites grown on the perimeter of the $\mathrm{Li}^{0}$ cathode. $\gamma=t_{\text {OFF }} / t_{O N}$ is the idle ratio.

Basic arguments clarify the physical meaning of the $t_{O N} \sim 1 \mathrm{~ms}$ time scale. The mean diffusive (MSD) displacement of $\mathrm{Li}^{+}$ions: $\mathrm{MSD}=\left(2 D_{+} t\right)^{1 / 2},\left(D_{+}\right.$is the experimental diffusion coefficient of $\mathrm{Li}^{+}$in $\mathrm{PC}$ ) defines the thickness of the depletion layers created in the vicinity of the 
cathode surface via Faradaic reduction of $\mathrm{Li}^{+}$that could be half-replenished via diffusion during $t$ rest periods. ${ }^{15}$ Notice that MSD is a function of time and only depends on a property of the system $\left(D_{+}\right)$, i.e. it is independent of operating conditions, such as current density. From the Einstein relationship: $D_{+}=\mu_{+}(R T / F)^{31}\left(\mu_{+}=\right.$is the mobility of $\mathrm{Li}^{+}$in $\mathrm{PC}$ ), the electric fields $|\mathrm{E}|_{\mathrm{c}}$ at which $\mathrm{Li}^{+}$electromigration displacements: $\mathrm{EMD}=\mu_{+}|\mathrm{E}|_{\mathrm{c}} t$, match MSD are given by equation ( $\mathrm{E}$ 2):

$$
|\mathrm{E}|_{\mathrm{c}}=(2 R \mathrm{~T} / F)^{1 / 2} \mu_{+}^{-1 / 2} t^{-1 / 2}
$$

Thus, with $(2 R T / F)=50 \mathrm{mV}$ at $300 \mathrm{~K}, \mu_{+}=1 \times 10^{-4} \mathrm{~cm}^{2} \mathrm{~V}^{-1} \mathrm{~s}^{-1}, t=1 \mathrm{~ms}$, we obtain $|\mathrm{E}|_{\mathrm{c}}=707 \mathrm{~V} \mathrm{~cm}^{-}$ 1 , which is considerably stronger than the homogeneous field between (flat) electrodes at the beginning of experiments: $|\mathrm{E}|_{0} \sim \mathrm{V}_{0} / L=9.4 \mathrm{~V} \mathrm{~cm}^{-1}$. However, cathode flatness and field homogeneity are destroyed upon the inception of dendrites, in whose sharp (i.e. large radii of curvature) tips local fields arise that are much stronger than $|\mathrm{E}|_{0}{ }^{15,32}$ Under such conditions, $\mathrm{Li}^{+}$ will preferentially migrate to the tips of advancing dendrites rather than to flat or concave sectors of the cathode surface. ${ }^{14-15,33-35}$ Furthermore, given the stochastic nature of dendrite propagation, one should expect a distribution of tip curvatures. Hence, the mean field condition: $E M D \leq M S D$, at specified $t_{O N}$ values may be realized by a subset of the population of dendrites. On sharper dendrites the inequality EMD > MSD will apply at the end of $t_{\text {ON }}$ pulses. Thus, larger $|\mathrm{E}|_{\mathrm{c}}$ values extend the EMD $\leq \mathrm{MSD}$ conditions to dendrites possessing sharper tips, i.e. to a larger set of dendrites that could be controlled by pulsing. Note, however, the weak $|\mathrm{E}|_{\mathrm{c}}$ $\propto \mu_{+}^{-1 / 2} \propto \eta^{-1 / 2}$ dependence on solvent viscosity $\eta$.

From this perspective, since $|\mathrm{E}|_{\mathrm{c}} \propto t^{-1 / 2}$, the application of longer charging pulses will increase the width of the depletion layers over a larger subset of dendrites to such an extent that such layers could not be replenished during rest periods, thereby magnifying the conditions favoring dendrite propagation. The preceding analysis suggests that shorter $t_{O N}$ periods should be increasingly beneficial. Could $t_{O N}$ be shortened indefinitely? The answer is no, because at sufficiently high pulsing frequencies charging will approach a DC regime. The transition from pulsed to DE charging takes place when $t_{O N}$ becomes shorter than the characteristic times of the transients associated with the capacitive polarization of electrochemical double layers upon 
application of a step voltage. A fundamental analysis of such transients ${ }^{36-40}$ revealed that the primary time scale for diffuse-charge dynamics is given by $\tau_{c}$, equation (E 3):

$$
\tau_{\mathrm{C}}=\lambda_{\mathrm{D}} L / D_{+}
$$

where $\lambda_{D}=\left(\varepsilon k_{B} T / 2 z^{2} e^{2} C_{0}\right)^{1 / 2}$ is the Debye screening length, $L$ the inter-electrode gap and $D^{+}$the $\mathrm{Li}^{+}$diffusion coefficient. In our system, for $\mathrm{C}_{0}=1 \mathrm{M} \mathrm{Li}^{+}$solutions in PC $(\varepsilon=65), D_{+}=2.58 \times 10^{-6}$ $\mathrm{cm}^{2} \mathrm{~s}^{-1}$, at $298 \mathrm{~K}$, we get: $\lambda_{\mathrm{D}}=0.27 \mathrm{~nm}$ and $\tau_{\mathrm{c}}=3.3 \mathrm{~ms}$. In physical terms, $\tau_{\mathrm{c}}=3.3 \mathrm{~ms}$ is the characteristic time for charging the capacitors associated with diffuse double layers. Capacitive charging implies the partial segregation of anions from cations, a phenomenon that increases the electric field close to the cathode surface and, therefore, triggers Faradaic deposition. Under $t_{O N}$ pulses increasingly shorter than $\tau_{c}$, most of the initial current will be capacitive, thereby implying that Faradaic currents will continue to circulate during the rest periods. In other words, the decreasing amplitude of polarization oscillations under trains of $t_{\text {ON }}$ pulses shorter than $\tau_{c}$ will gradually converge to steady DC.

In summary, shorter $t_{O N}$ pulses are increasingly beneficial in inhibiting dendrite propagation, but they are loosely bound by the condition $t_{O N} \geq \tau_{c}$. The underlying reason is that shorter $t_{O N}$ pulses will inhibit dendrite at earlier propagation stages, where the curvature of most dendrite tips has not reached the magnitude at which the local electric fields lead to the EMD > MSD runaway condition. Notice that the stage at which dendrite propagation can be controlled by pulsing relates to the curvature of tip dendrites, which is a morphological condition and therefore independent of current density. Higher current densities, however, will shorten the induction period preceding dendrite nucleation. ${ }^{34}$

These ideas were cast and tested in a coarse-grain Monte Carlo model that, in accord with the preceding arguments, deals explicitly with ion diffusion, electromigration and deposition. It should be emphasized that our model is more realistic than those previously reported ${ }^{9}$ because $^{2}$ it takes into account the important fact that dendritic growth should be critically dependent on the strong electric fields that develop about the dendrites tips upon charging. ${ }^{41}$ In fact, the key role of electromigration in dendrite propagation has been recently demonstrated by the smooth $\mathrm{Li}^{0}$ cathode surfaces produced in the presence of low concentrations of non-reducible cations, such as $\mathrm{Cs}^{+}$that, by preferentially accumulating on dendrite tips, neutralize the local 
electric fields and deflect $\mathrm{Li}^{+}$toward the flat cathode regions. ${ }^{3}$ Thus, both the population of electroactive $\mathrm{Li}^{+}$species and the electric vector field within the partially depleted, polarized double layers surrounding the cathode is established from the competition of ion diffusion with electromigration rather than with $\mathrm{Li}^{+}$deposition. Given the typically small overpotentials for metal ion reduction on metallic electrodes, ${ }^{31}$ we consider that the effect of the applied external voltage on dendrite growth operates via the enhancement of $\mathrm{Li}^{+}$migration rather than $\mathrm{Li}^{+}$ reduction. In our model dendrite nucleation is a purely statistical phenomenon, i.e., nucleation occurs because there is a finite probability that two or more $\mathrm{Li}^{+}$ions are successively reduced at a given spot on the cathode surface. Once a dendrite appears, a powerful positive feedback mechanism sets in. The enhanced electric field at the tip of the sharp dendrite attracts $\mathrm{Li}^{+}$ions faster, thereby accelerating dendrite propagation and depleting the solution of $\mathrm{Li}^{+}$in its vicinity. Thus, the anisotropic concentration gradients observed nearby growing dendrites may be a consequence of the statistical onset of dendrites. In other words, we consider that simultaneity does not imply causality, and that the fast depletion of $\mathrm{Li}^{+}$upon dendrite inception is an effect than a cause of dendrite nucleation. We wish to emphasize, however, that we are aware our arguments and modeling effort do not exclude alternative proposals to account for dendrite nucleation. Furthermore, we also want to underscore the fact out that the validity of one view or another on the mechanism of dendrite nucleation may not affect our interpretation that the effects of pulsing on dendrite propagation arise from the competition between ion diffusion and electromigration once dendrites appear. Due to the very high computational cost of atomistic modeling, we simulate the processes taking place a domain that is smaller than the actual cell. Its actual dimensions approximately correspond to the depth of a depletion boundary layer at the cathode. Calculations are based on the scaled parameters shown in Table 1. Scaling was guided by the following considerations. Because our calculations aim at reproducing the frequency response of our experiments, simulation time was set to real time. The length scale, however, is dictated by computational cost. Thus, if diffusional displacements in real time would not exceed the size of our domain $(L=16.7 \mathrm{~nm}$, Table 1$)$ the diffusion coefficient should be scaled accordingly. The adopted $D_{+}=1.40 \times 10^{-10} \mathrm{~cm}^{2} / \mathrm{s}$ value in fact leads to MSD $\sim 0.3 L$ after $1 \mathrm{~ms}$. A voltage difference of $\left(V_{\text {top }}-V_{\text {cathode }}\right)=14.7 \mathrm{mV}$ between the 
electrodes separated by $L=16.7 \mathrm{~nm}$ generates electric fields in which electromigration is competitive with diffusion. The implemented two-dimensional Monte Carlo algorithm calculates the trajectories of individual $\mathrm{Li}^{+}$ions via random diffusion and electro- migration under time and position-dependent electric fields $\overrightarrow{E(x, y, t)}$.

Table 1 -Parameters used in the Monte Carlo calculations

\begin{tabular}{|l|l|}
\hline Domain size $L$ & $16.7 \mathrm{~nm} \times 16.7 \mathrm{~nm}$ \\
\hline$\Delta \mathrm{t}$ (integration step) & $1 \mu \mathrm{s}$ \\
\hline $\mathrm{V}_{\text {cathode }}$ & $0 \mathrm{~V}$ \\
\hline $\mathrm{V}_{\text {top }}$ & $47.4 \mathrm{mV}$ \\
\hline $\mathrm{D}_{+}\left(\mathrm{Li}^{+}\right.$diffusion coefficient) & $1.40 \times 10^{-10} \mathrm{~cm}^{2} / \mathrm{s}$ \\
\hline$\mu_{+}\left(\mathrm{Li}^{+}\right.$mobility) & $5.54 \times 10^{-9} \mathrm{~cm}^{2} / \mathrm{Vs}$ \\
\hline $\mathrm{Li}^{+}$radius & $1.19 \AA$ \\
\hline Free $\mathrm{Li}^{+}$ions & 50 \\
\hline Maximum $\mathrm{Li}^{0}$ atoms & 600 \\
\hline
\end{tabular}

By assuming that $\mathrm{Li}^{+}$ions reach stationary velocities instantaneously, their mean displacements are given by:

$$
\overrightarrow{\boldsymbol{r}_{l}}(t+\Delta t)-\overrightarrow{\boldsymbol{r}_{l}}(t)=\sqrt{2 D_{+} \Delta t} \overrightarrow{\boldsymbol{g}}+\mu_{+} \overrightarrow{\boldsymbol{E}} \Delta t
$$

The first and second terms in the RHS of E3 are the mean displacements due to ionic diffusion and electromigration, respectively. $\vec{g}$ is a normalized 2D vector representing random motion via diffusion, $\Delta t$ is the computational time interval, and $\underline{\boldsymbol{E}}$ is the electric field vector. By normalizing displacements relative to the inter-electrode separation, $L$, (E 4) transforms into (E 5):

$$
\vec{\xi}(t+\Delta t)=\overrightarrow{\boldsymbol{\xi}}(t)+\theta \overrightarrow{\boldsymbol{g}}+\overrightarrow{\boldsymbol{\eta}}
$$

Dendrite lengths $\lambda_{\mathrm{i}}$ were evaluated as their height $\alpha_{i}(t)$ above the surface of the electrode:

$$
\lambda_{i}(t)=\max _{k=1: n} \vec{\xi}_{k}(t) \cdot \boldsymbol{j}
$$

$\boldsymbol{j}$ is the unit vector normal to the surface of the electrode and $n$ is the total number of lithium atoms incorporated into the dendrite.

By using the Einstein relationship above, the equation of motion becomes:

$$
\vec{r}(t+\Delta t)-\vec{r}(t)=\sqrt{2 D_{+} \Delta t} \hat{g}+\frac{F}{R T} D_{+} \Delta t \vec{E}
$$

a function of $D_{+} \Delta t$. 
By neglecting electrostatic ion-ion interactions, given that they are effectively screened since $\lambda_{D}=0.27 \mathrm{~nm}$ is smaller than the average interionic separation $R_{i, j}=1.2 \mathrm{~nm}, \overrightarrow{\boldsymbol{E}(\boldsymbol{x}, \boldsymbol{y}, \boldsymbol{t})}$ is computed using Laplace's equation:

$$
\frac{\partial^{2} \phi}{\partial x^{2}}+\frac{\partial^{2} \phi}{\partial y^{2}}=0
$$

Thus, the electric field is determined by the evolving geometry of the equipotential dendritic cathode. In reality, the concentration gradients that develop in the depleted boundary layers would lead to even greater electric field enhancements than reported herein. The inclusion of ion-ion interactions and charge imbalances in Monte Carlo calculations would be too costly computationally. We consider, however, that the inclusion of a variable electric field represents a significant advance over previous models. ${ }^{9}$

Calculated dendrite heights were quantified by dividing the $\mathrm{x}$-axis (parallel to the surface of the cathode) in four sectors. Here, 'dendrite height' in each sector is the height of the $\mathrm{Li}^{0}$ atoms furthest from the electrode. To ensure good statistics, each simulation was run 100 times, for a total of 400 measurements per data point. The key experimental result, i.e., that longer $t_{\text {OFF }}$ rest periods are significantly more effective in reducing $\alpha$ after $t_{O N}=1 \mathrm{~ms}$ than $t_{O N}=20 \mathrm{~ms}$ charging pulses, is clearly confirmed by calculations (Fig. 2 and Fig. S1). Fig. 3 displays the results of sample simulations. Metallic dendrites grow with random morphologies into equipotential structures held at $V=0$ Volts, thereby perturbing the uniform electric field prevailing at the beginning of the experiments. The high-curvature dendrite tips act as powerful attractors for the electric field, which by accelerating $\mathrm{Li}^{+}$toward their surfaces deplete the electrolyte, further enhancing the field intensity. This strong positive feedback mechanism has its counterpart in the electrolyte regions surrounded by dendrites. The electric field therein nearly vanishes as a consequence of being surrounded by concave equipotential surfaces. ${ }^{31}$ The key feature is that ion displacements from electromigration are proportional to $\tau_{\mathrm{ON}}$, whereas diffusive ones increase as $\tau_{\mathrm{ON}}^{1 / 2}$. Above some critical $\tau_{\mathrm{ON}}$ value, the depth of the deplete layers will increase to the point at which they could not be replenished during the ensuing rest periods regardless of their duration.

These phenomena are visualized from the computational results shown in Figs. 3-6. Fig. 4 displays the dendrite morphologies created by pulsing at various $\gamma^{\prime}$ s. Calculations for longer $t_{\text {off }}$ 
values show marginal improvements, since $\partial\left[\mathrm{Li}^{+}\right] / \partial y$ gradients remain largely unaffected in simulations for $\gamma>3$. Fig. 5 shows typical morphologies of dendrites consisting of a given number of deposited $\mathrm{Li}^{0}$.
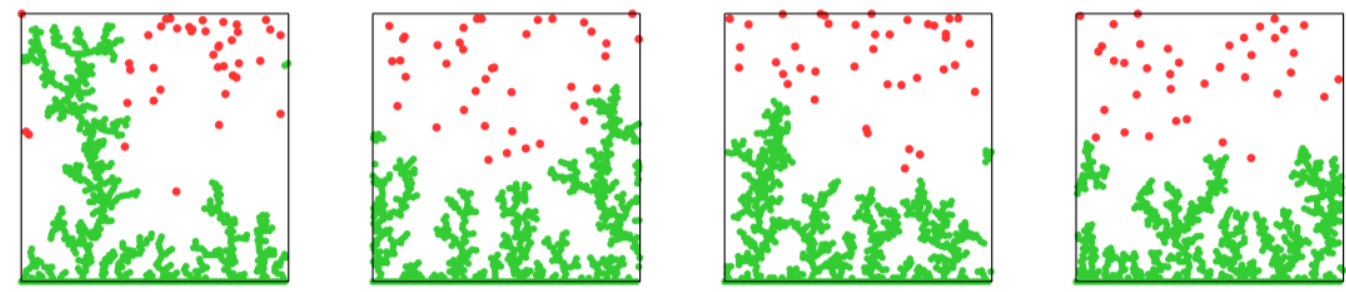

Fig. 3 - Left to right: dendrite morphologies for DC charging, charging with $t_{\mathrm{ON}}=1 \mathrm{~ms}$ pulses at $\gamma=t_{\mathrm{OFF}} / \mathrm{t}_{\mathrm{ON}}=1,2$ and 3. Green dots: $\mathrm{Li}^{0}$. Red dots: $\mathrm{Li}^{+}$.

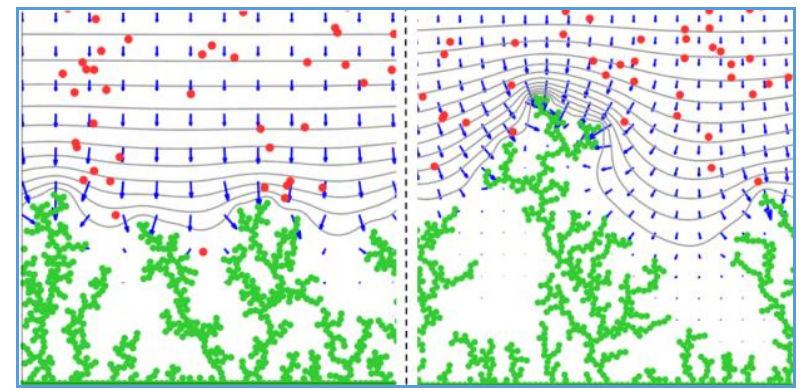

Fig. 4 - Simulations for charging with $\mathrm{t}_{\mathrm{ON}}=1 \mathrm{~ms}$ (left) and $\mathrm{t}_{\mathrm{ON}}=20 \mathrm{~ms}$ (right) at $\gamma=\mathrm{t}_{\mathrm{OFF}} / \mathrm{t}_{\mathrm{ON}}=3$. Green dots: $\mathrm{Li}^{0}$. Red dots: $\mathrm{Li}^{+}$. Gray lines: equipotential contours. Blue vectors: the electric field.

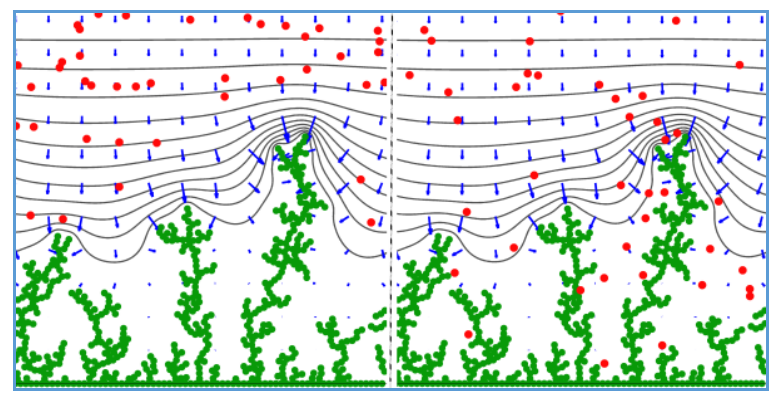

Fig. 5 - Simulations for charging with $t_{\mathrm{ON}}=1 \mathrm{~ms}, \gamma=1$ pulses. Left: after a charging pulse. Right: at the end of the successive rest period (right). Green dots: LiO. Red dots: Li+. Gray lines: equipotential contours. Blue vectors: the electric field. 


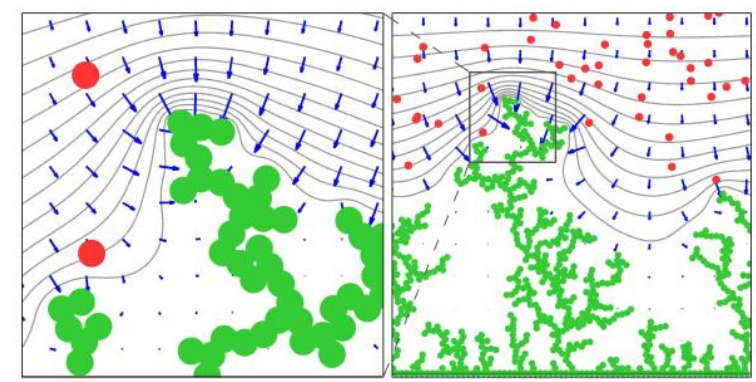

Fig. 6 - Zooming in the tip of the leading dendrite produced by charging with $t_{O N}=20 \mathrm{~ms}, \gamma=t_{O F F} / t_{O N}=3$ pulses for $243 \mathrm{~ms}$, i.e., at the end of simulation time. Green dots: LiO. Red dots: Li+. Gray lines: equipotential contours. Blue vectors: the electric field.

Summing up, we have demonstrated that (1) by charging our lithium metal cell with $t_{O N}=1$ $\mathrm{ms}, \gamma=t_{O F F} / t_{O N}=3$ pulse trains the average dendrite length $\alpha$ is significantly reduced (by $\sim 70$ \%) relative to DC charging, (2) such pulses are nearly optimal for dendrite inhibition because they are commensurate with the relaxation time $\tau_{\mathrm{c}} \sim 3 \mathrm{~ms}$ for the diffusive charging of the electrochemical double layers in our system. Monte Carlo simulations dealing explicitly with lithium ion diffusion, electromigration in time-dependent electric fields and deposition at the cathode were able to reproduce the trends on the experimental effects of the $t_{O N}$ charging and $t_{\text {OFF }}$ rest periods on $\alpha$. Further work along these lines is underway.

Acknowledgments - We would like to thank undergraduate student Alejandro Sánchez for help in making cell parts. This work is sponsored by Bill and Melinda Gates Foundation Grant No. OPP1069500 on environmental sustainability, and in part by Bosch Energy Research Network Grant No. 13.01.CC11.

Supporting information - Additional data, experimental and calculational details, is available free of charge via the internet at http://pubs.acs.org/

\section{References}

(1) Xu, W.; Wang, J.; Ding, F.; Chen, X.; Nasybulin, D.; Zhang, Y.; Zhang, J. G. Lithium Metal Anodes for Rechargeable Batteries. Energy Environ. Sci 2014.

(2) Armand, M.; Tarascon, J. M. Building Better Batteries. Nature 2008, 451 (7179), 652-657.

(3) Ding, F.; Xu, W.; Graff, G. L.; Zhang, J.; Sushko, M.; Chen, X.; Shao, Y.; Engelhard, M. H.; Nie, Z.; Xiao, J. Dendrite-Free Lithium Deposition Via Self-Healing Electrostatic Shield Mechanism. Journal of the American Chemical Society 2013.

(4) Chandrashekar, S.; Trease, N. M.; Chang, H. J.; Du, L.-S.; Grey, C. P.; Jerschow, A. 7li Mri of Li Batteries Reveals Location of Microstructural Lithium. Nature Materials 2012, 11 (4), 311-315.

(5) Harry, K. J.; Hallinan, D. T.; Parkinson, D. Y.; MacDowell, A. A.; Balsara, N. P. Detection of Subsurface Structures Underneath Dendrites Formed on Cycled Lithium Metal Electrodes. Nat Mater 2014, 13 (1), 69-73. 
(6) Rugolo, J.; Aziz, M. J. Electricity Storage for Intermittent Renewable Sources. Energy \& Environmental Science 2012, 5 (5), 7151-7160.

(7) Huskinson, B.; Marshak, M. P.; Suh, C.; Er, S.; Gerhardt, M. R.; Galvin, C. J.; Chen, X.; Aspuru-Guzik, A.; Gordon, R. G.; J. Aziz, M. J. A Metal-Free Organic-Inorganic Aqueous Flow Battery. Nature 2014, 505, 195-198.

(8) Goodenough, J. B. Rechargeable Batteries: Challenges Old and New. J. Solid State Electrochem. 2012, 16 (6), 2019-2029.

(9) Mayers, M. Z.; Kaminski, J. W.; Miller III, T. F. Suppression of Dendrite Formation Via Pulse Charging in Rechargeable Lithium Metal Batteries. The Journal of Physical Chemistry C 2012, 116 (50), 2621426221.

(10) Nishida, T.; Nishikawa, K.; Rosso, M.; Fukunaka, Y. Optical Observation of Li Dendrite Growth in Ionic Liquid. Electrochim. Acta 2013.

(11) Williard, N.; He, W.; Hendricks, C.; Pecht, M. Lessons Learned from the 787 Dreamliner Issue on Lithium-Ion Battery Reliability. Energies 2013, 6 (9), 4682-4695.

(12) Xu, K. Nonaqueous Liquid Electrolytes for Lithium-Based Rechargeable Batteries. Chemical ReviewsColumbus 2004, 104 (10), 4303-4418.

(13) Nishikawa, K.; Mori, T.; Nishida, T.; Fukunaka, Y.; Rosso, M.; Homma, T. In Situ Observation of Dendrite Growth of Electrodeposited Li Metal. J. Electrochem. Soc. 2010, 157 (11), A1212-A1217.

(14) Fleury, V. Branched Fractal Patterns in Non-Equilibrium Electrochemical Deposition from Oscillatory Nucleation and Growth. Nature 1997, 390 (6656), 145-148.

(15) Chazalviel, J. N. Electrochemical Aspects of the Generation of Ramified Metallic Electrodeposits. Phys. Rev. A 1990, 42 (12), 7355-7367.

(16) Ely, D. R.; Garcia, R. E. Heterogeneous Nucleation and Growth of Lithium Electrodeposits on Negative Electrodes. J. Electrochem. Soc. 2013, 160 (4), A662-A668.

(17) Akolkar, R. Mathematical Model of the Dendritic Growth During Lithium Electrodeposition. J. Power Sources 2013, 232, 23-28.

(18) Brissot, C.; Rosso, M.; Chazalviel, J. N.; Lascaud, S. In Situ Concentration Cartography in the Neighborhood of Dendrites Growing in Lithium/Polymer-Electrolyte/Lithium Cells. J. Electrochem. Soc. 1999, 146 (12), 4393-4400.

(19) F. Orsini, A. D. P., B. Beaudoin, J.M. Tarascon, M. Trentin, N. Langenhuisen, E.D. Beer, P. Notten. In Situ Scanning Electron Microscopy (Sem) Observation of Interfaces with Plastic Lithium Batteries. J. Power Sources 1998, 76, 19-29.

(20) Monroe, C.; Newman, J. Dendrite Growth in Lithium/Polymer Systems - a Propagation Model for Liquid Electrolytes under Galvanostatic Conditions. J. Electrochem. Soc. 2003, 150 (10), A1377-A1384.

(21) Crowther, O.; West, A. C. Effect of Electrolyte Composition on Lithium Dendrite Growth. J. Electrochem. Soc. 2008, 155 (11), A806-A811.

(22) Howlett, P. C.; MacFarlane, D. R.; Hollenkamp, A. F. A Sealed Optical Cell for the Study of LithiumElectrode Electrolyte Interfaces. J. Power Sources 2003, 114 (2), 277-284.

(23) Schweikert, N.; Hofmann, A.; Schulz, M.; Scheuermann, M.; Boles, S. T.; Hanemann, T.; Hahn, H.; Indris, S. Suppressed Lithium Dendrite Growth in Lithium Batteries Using lonic Liquid Electrolytes: Investigation by Electrochemical Impedance Spectroscopy, Scanning Electron Microscopy, and in Situ Li7 Nuclear Magnetic Resonance Spectroscopy. J. Power Sources 2013, 228, 237-243.

(24) Basile, A.; Hollenkamp, A. F.; Bhatt, A. I.; O'Mullane, A. P. Extensive Charge-Discharge Cycling of Lithium Metal Electrodes Achieved Using Ionic Liquid Electrolytes. Electrochemistry Communications 2012.

(25) Rosso, M.; Gobron, T.; Brissot, C.; Chazalviel, J. N.; Lascaud, S. Onset of Dendritic Growth in Lithium/Polymer Cells. J. Power Sources 2001, 97-8, 804-806. 
(26) Seong, I. W.; Hong, C. H.; Kim, B. K.; Yoon, W. Y. The Effects of Current Density and Amount of Discharge on Dendrite Formation in the Lithium Powder Anode Electrode. J. Power Sources 2008, 178 (2), 769-773.

(27) Stone, G.; Mullin, S.; Teran, A.; Hallinan, D.; Minor, A.; Hexemer, A.; Balsara, N. Resolution of the Modulus Versus Adhesion Dilemma in Solid Polymer Electrolytes for Rechargeable Lithium Metal Batteries. J. Electrochem. Soc. 2012, 159 (3), A222-A227.

(28) Bhattacharyya, R.; Key, B.; Chen, H. L.; Best, A. S.; Hollenkamp, A. F.; Grey, C. P. In Situ Nmr Observation of the Formation of Metallic Lithium Microstructures in Lithium Batteries. Nature Materials 2010, 9 (6), 504-510.

(29) Benjacob, E.; Garik, P. The Formation of Patterns in Nonequilibrium Growth. Nature 1990, 343 (6258), 523-530.

(30) Huggins, R. Advanced Batteries: Material Science Aspects. Springer: New York, 2008.

(31) Bard, A. J.; Faulkner, L. R. Electrochemical Methods: Fundamentals and Applications.1980, 2 New York: Wiley, 1980.

(32) Jackson, J. D. Classical Electrodynamics. Wiley: New York 1999.

(33) Roy, S. Formation of Dual Diffusion Layer by Pulsing Currents. Ind. Eng. Chem. Res. 2012, 51 (4), 1756-1760.

(34) Diggle, J.; Despic, A.; Bockris, J. M. The Mechanism of the Dendritic Electrocrystallization of Zinc. J. Electrochem. Soc. 1969, 116 (11), 1503-1514.

(35) Wang, M.; Ming, N. B. Concentration Field Oscillation in Front of a Dendrite Tip in Electrochemical Deposition. Phys. Rev. A 1992, 45 (4), 2493-2498.

(36) Bazant, M. Z.; Thornton, K.; Ajdari, A. Diffuse-Charge Dynamics in Electrochemical Systems. Phys. Rev. E 2004, 70 (2).

(37) Norton, J. D.; White, H. S.; Feldberg, S. W. Effect of the Electrical Double-Layer on Voltametry at Microelectrodes. Journal of Physical Chemistry 1990, 94 (17), 6772-6780.

(38) Alexe-lonescu, A. L.; Barbero, G.; Bianco, S.; Cicero, G.; Pirri, C. F. Electrical Response of Electrolytic Cells Limited by Different Types of Electrodes. J. Electroanal. Chem. 2012, 669, 21-27.

(39) Macdonald, J. R. Theory of Ac Space-Charge Polarization Effects in Photoconductors, Semiconductors and Electrolytes. Physical Review 1953, 92 (1), 4-17.

(40) Hossain, R.; Adamiak, K. Dynamic Properties of the Electric Double Layer in Electrolytes. Journal of Electrostatics 2013, 71 (5), 829-838.

(41) Zhong, X. K.; Guo, X. P.; Qiu, Y. B.; Chen, Z. Y.; Zhang, G. A. In Situ Study the Electrochemical Migration of Tin under Unipolar Square Wave Electric Field. J. Electrochem. Soc. 2013, 160 (11), D495D500. 